\title{
REVISTA NUEVA: EL VALOR DE LA CULTURA
}

\section{NEW MAGAZINE: THE VALUE OF CULTURE}

\section{AUTORA}

\section{Isabel Tajahuerce Ángel}

Facultad de Ciencias de la Información. Universidad Complutense de Madrid.

isabel.tajahuerce@yahoo.es

\section{RESUMEN}

En los años siguientes a la construcción del Canal de Panamá la Revista Nueva fue una de las publicaciones culturales más importantes. Su objetivo era resaltar una conciencia nacional de carácter optimista. En este número de la revista se remarca además la importancia que se le concedía a la educación y a los estudios pedagógicos, así como que también recoge el carácter revolucionario que se respiraba en el panorama internacional.

\section{PALABRAS CLAVE}

Revista Nueva - Educación - Cultura - Pensamiento revolucionario.

\section{ABSTRACT}

In the years following the construction of the Panama Canal New Magazine was one of the most important cultural publications. Its aim was to highlight national awareness optimistic character. In this issue of the magazine also highlights the 
importance he attached to education and pedagogical studies, and also reflects the revolutionary character that was present in the international arena.

\section{KEY WORDS}

Revista Nueva - Educucation - Culture - Revolutionary thought

\section{ÍNDICE}

1. Optimismo y conciencia nacional

2. Educación y cultura.

3. Una tribuna abierta.

La Revista Nueva (1916-1919) fue una de las publicaciones culturales más importantes de los críticos años que siguieron a la construcción del canal. Su objetivo era "informar del movimiento intelectual del pais", fomentar el desarrollo de una verdadera conciencia nacional y difundir la cultura, sin pensar en los beneficios económicos. Sus editores fueron dos hombres que dedicaron parte de su vida a la educación y que colaboraron en muchos otros proyectos de interés para el país: José Dolores Moscote y Octavio Méndez Pereira.

José Dolores Moscote fue un intelectual "en el más noble sentido de la palabra", como dijo Rodrigo Miró Grimaldo al ocupar su lugar en la Academia Panameña de la lengua, y realizó una interesante labor docente tanto en el Instituto Nacional como en la Universidad, además de dedicarse al estudio del constitucionalismo buscando "modos de convivencia acordes con nuestra idiosincrasia". En cuanto a Octavio Méndez Pereira fue el impulsor de la Universidad de Panamá (1935) y su primer rector, para lo cual contará con la inestimable colaboración de J. Dolores Moscote y

\footnotetext{
${ }^{1}$ MIRÓ GRIMALDO, Rodrigo: "Patria en su contexto histórico (Discurso de incorporación como individuo de número de la Academia Panameña de la Lengua, en acto celebrado el 25 de octubre de 1978)" en Revista Tareas. Panamá, mayo-agosto 1996.págs. 7-28.
} 
varios colaboradores de La Revista Nueva, como José Daniel Crespo autor de varios artículos sobre pedagogía.

\section{1. Optimismo y conciencia nacional.}

El número de la revista que hemos analizado es el que corresponde a enero de 1917. Los textos publicados a lo largo de sus 80 páginas abordan distintos temas, pero hay siempre una prioridad: resaltar la necesidad del desarrollo de una conciencia nacional desde una perspectiva optimista. Para ello es necesario restar importancia (aunque ello no implique ignorar la realidad) a la política de los Estados Unidos en la República desde la firma del Tratado Hay-Bunau Varilla en 1903, una política "agresiva y colonialista", como señala Víctor Ávila, que en 1904 obligó al gobierno panameño a disolver su ejército nacional y a plantear más tarde la necesidad de desarmar a la policía, medida que fue impuesta en 1916 "alegando en esa oportunidad que con frecuencia surgían incidentes entre policías panameños y soldados norteamericanos" ${ }^{2}$.

Los optimistas (aunque saben que el sistema no es perfecto) creen que el país va por buen camino. Tal vez influya en ese optimismo la prosperidad económica de aquellos años. Marco A. Gandásegui comenta que la nueva democracia panameña de "ciudadanos" hallaba su apoyo por un lado en la masa de pequeños agricultores agrícolas que se "insertaron rápidamente en el dinámico mercado generado por la construcción del Canal de Panamá" y por otro en "la prosperidad comercial creada por las demandas de la primera guerra mundial"3. Es, por lo tanto, un buen momento y esto se refleja en la forma de afrontar el futuro por parte de los sectores liberales.

\footnotetext{
2 ÁVILA, Víctor (1998): Panamá: luchas sociales y afirmación nacional. Centro de Estudios Latinoamericanos "Justo Arosemena". Panamá, pág.57.

3 GANDÁSEgUI, Marco A. (1998): La democracia en Panamá. Centro de Estudios Latinoamericanos "Justo Arosemena". Panamá, pág.19.
} 
De la lectura de los artículos y discursos publicados en La Revista Nueva se deduce que los intelectuales panameños estaban claramente divididos en "optimistas" y "pesimistas". Es especialmente interesante el discurso pronunciado por el jurista Ricardo J. Alfaro en el Instituto Nacional y con el que se inicia este segundo tomo en 1917, pues aporta muchos datos sobre los temores y esperanzas de los liberales en aquellos años.

Alfaro recurre a la Historia y a las Relaciones Internacionales para demostrar que siempre han existido países dominados por otros, que la lucha por la independencia es dura y que al fin y al cabo ahora Panamá está mejor de lo que ha estado nunca. Define el patriotismo como "el amor a la tierra en que nacimos, la obligación de servirla y de contribuir a su engrandecimiento", y concluye que la raíz de ese patriotismo "se halla en la conciencia de la nacionalidad: en la certeza, la satisfacción, el orgullo de pertenecer a una comunidad nacional". Destaca, además la "solidaridad panamericana" a partir de la doctrina Monroe porque:

\begin{abstract}
"esa doctrina significa que la América libre no es campo abierto a la colonización y que no es lícito a ninguna potencia -ni europea ni americana- arrebatar su libertad a los países que han logrado conquistarla" ${ }^{4}$.
\end{abstract}

Quien con el tiempo será uno de los encargados de elaborar un proyecto de Constitución afirma que durante el siglo XIX el Istmo era ya una Nación porque en el exterior "un hijo del Istmo" se olvidaba siempre de su nacionalidad y decía" soy panameño". Desde el 3 de Noviembre de 1903 Panamá es un Estado, por lo tanto hay muchos motivos para mostrarse optimista aunque quede mucho camino por andar:

\footnotetext{
${ }^{4}$ ALFARO, Ricardo F.: “El sentimiento nacional (Discurso patriótico pronunciado por el señor don Ricardo J. Alfaro el 3 de Noviembre pasado, en el aula Máxima del Instituto Nacional)" en La Revista Nueva. Panamá, enero 1917, pp. 1-12.
} 
“! Nación y Estado! ¿Os habéis detenido a meditar el alcance trascendental de estos dos conceptos? ¡Los istmeños libres y con gobierno propio! El sueño de Tomás Herrera y de Justo Arosemena convertido en bella realidad. La aspiración suprema de tantos otros pueblos la tierra, ricos, populosos, civilizados, desarrollados por completo y que, sin embargo, carecen de personería internacional, la hemos alcanzado nosotros, tan pobres, tan débiles, tan pequeños. ¿Cómo dejar que se pierda ese tesoro inapreciable? cómo no estremecernos de placer y de orgullo cuando vibran en el aire las notas marciales de la canción nacional y flota llena de gracia la bandera de las dos estrellas?. Creo señores, que los istmeños no solamente debemos sentirnos satisfechos de nuestra propia suerte sino que estamos en la obligación de mantenerla y de mejorarla" ${ }^{5}$.

Los "pesimistas" no ven las cosas de la misma manera. Ellos están convencidos e intentan convencer con sus argumentos del peligro de muerte que acecha a la nacionalidad panameña, un peligro que viene de EEUU. No les falta razón cuando dicen, según Alfaro:

"Esa potencia es imperialista y absorbente; aspira a la hegemonía continental $y$ en sus nuevas expansiones territoriales es lo más natural que comiencen por Panamá. ¿No veis cuántas exigencias e imposiciones hacen sufrir a la República?¿No observáis la conducta de esa potencia en otras partes?. En Nicaragua (...) Haití (...) Santo Domingo (...) Cuba, lo mismo que Panamá. Vive en vida política bajo la amenaza perenne de la intervención armada. Y luego ¿no es un hecho que nosotros no podemos hacer en nuestro suelo todo lo que

\footnotetext{
5 Ibidem, pág. 9.
} 
queremos? ¿Nuestra soberanía no está limitada por la voluntad de esa potencia del Norte?"6.

Pero, aunque esto sea cierto Alfaro lo justifica para no "destruir el optimismo patriótico" afirmando que no hay "una sola nación en el mundo cuya soberanía no encuentre una limitación en la solidaridad internacional". Ninguna nación, y menos aún las pequeñas, puede hacer lo que quiera, y para evitar que se justifique una intervención hay que evitar el "desorden". Intenta convencer de que se acepte la situación porque nunca Panamá estuvo mejor y los pesimistas no ayudan nada con sus críticas, es mejor luchar de otra manera, demostrar hasta que punto los panameños están preparados para gobernarse:

“(..) si en nuestra conducta interior y exterior observamos la cordura, el tino y el tacto que debe caracterizar a un Gobierno serio y honrado, la República perdurará sin ser injustamente atacada, porque lo que es respetable infunde siempre respeto" ${ }^{\prime 7}$.

Pero no sólo es optimista Ricardo F. Alfaro, muchos otros juristas, políticos, profesores o escritores del momento lo son también, y en este número de La Revista Nueva domina esta visión de la realidad panameña. Así Ascanio Carles en el discurso que pronuncia a raíz del artículo 3o de la Ley 33 de 1912 defiende una postura similar cuando afirma:

"Nuestra patria nos ofrece hoy ese día que en su historia puso fin a sus dolores y lágrimas, y señaló la fecha de un despertar glorioso a la confianza optimista del futuro, brumoso aún para algunos

\footnotetext{
${ }^{6}$ Ibidem, págs.9-10.

7 Ibidem, pág. 12 .
} 
desconfiados que no han logrado todavía convencerse de que el menor es el preferible de los males"8.

No hay que ignorar los peligros, pero:

"(...) de todo el escepticismo que la experiencia nos enseña a observar en la vida, nuestra visión del porvenir debe ser optimista, por lo mucho que nos hemos acercado a su lejano horizonte" ${ }^{\text {. }}$.

También se muestra optimista Samuel Lewis en su conferencia sobre economía política al hablar del "valor" y el "precio". Basándose en las definiciones de autores como J. Stuart Mill, A. Smith o Gide, llega a la conclusión de que el valor del Istmo es elevadísimo por su posición privilegiada que permite establecer la comunicación entre dos océanos, por eso a lo largo de la historia se han estudiado las posibilidades que ofrecía:

"Es la historia la que se encarga de establecer, después de tres centurias de continua prueba, que las tierras del Istmo son objeto de deseabilidad en grado extraordinario para llenar necesidad imperiosa de la humanidad, y es a esa misma Historia a la que le toca poner de relieve la marcada rareza de territorio semejante, capaz de facilitar el cumplimiento de tan elevada misión. De allí que, aplicando a su valor inmutable el cartabón señalado por la deseabilidad y la rareza propias en ellas, sea forzoso declarar que las tierras del Istmo son de las más valiosas de la América" ${ }^{10}$.

\footnotetext{
8 ASCANIO, Carles (1997): “Discurso pronunciado en la sesión solemne del Consejo Municipal de Penomé, el día 3 de Noviembre de 1916, por Don Ascanio Carles“ en La Revista Nueva, Panamá, enero 1917. pág.50.

9 Ibidem, pág. 52 .

${ }^{10}$ LEWIS, Samuel: “La propiedad agraria, su valor y su precio. (Conferencia del señor don Samuel Lewis en el Aula Máxima del Instituto Nacional)"en La Revista Nueva. Panamá, enero 1917, pág. 19.
} 
El problema para Lewis es que el precio de esas tierras tan valiosas ha estado sujeto a constantes cambios. Las convulsiones políticas y sociales han impedido a lo largo de los siglos que se mantuviese el derecho de propiedad. Pero el 3 de noviembre de 1903 "nació robusto y vigoroso el derecho de propiedad de los istmeños" y desde entonces las cosas han cambiado considerablemente porque al respetarse el derecho de propiedad las tierras han adquirido valor.

La paz es fundamental para mantener el derecho de propiedad, derecho gracias al cual entran "en juego las dos palancas del precio: la oferta y la demanda". El precio de las tierras en ese momento es alto porque muchos factores han contribuido a que así sea, si se mantiene la paz y se respetan los derechos todo puede mejorar. El futuro no obstante queda en el aire:

"En atención a los principios enunciados, tenemos, para concluir, que el precio de nuestras tierras va en aumento y seguirá asi hasta que se cumpla una de las siguientes circunstancias: que el precio fluctuante de ellas coincida con su valor inmutable, o que el precio llegue a tal altura que anule la demanda"11.

\section{2. Educación y cultura.}

Muchos de los textos publicados en este y otros números de la revista son conferencias pronunciadas en el Instituto Nacional con el que tanto Octavio Méndez Pereira como José Dolores Moscote mantienen una estrecha relación, al igual que otros intelectuales panameños que soñaban con que la educación llegase al pueblo panameño para construir así un país fuerte, un país preparado y culto. Por eso, escribe Víctor Ávila, las "corrientes más progresistas del liberalismo panameño emprendieron la ingente empresa de dotar a la república de una infraestructura

11 Ibidem, pág.30. 
educativa"12, fruto de ello fue la creación de varios centros de enseñanza como Artes y Oficios, la Escuela Profesional y el citado Instituto Nacional que será siempre un centro de debate y discusión en el que habrá un lugar para las distintas opiniones. La misma inquietud cultural llevó que se creasen una serie de publicaciones de gran calidad como El Heraldo del Istmo (1904-1906), Nuevos Ritos (1907-1915), Cuasimodo (1919-1920) y La Revista Nueva (1916-1919), todas ellas revistas literarias con un fuerte contenido social que al igual que el Instituto Nacional se convirtieron en una tribuna abierta en la que con un espíritu crítico las figuras más relevantes de la cultura panameña del momento expresaron sus opiniones.

El valor que se daba a la educación se resalta en el discurso ya citado de Ricardo F. Alfaro cuando comenta su vinculación como profesor con el Instituto Nacional, aunque en ese momento se encuentre apartado de la docencia por su dedicación a otras tareas también importantes para el país:

"Yo siento la nostalgia de la clase. Una voz secreta y misteriosa me atrae con fuerza hacia el aula, santuario de la civilización donde el alma halla las más gratas expansiones. Allí se encuentran únicamente cerebros y corazones juveniles, repletos de los más nobles sentimientos y anhelos."

$Y$ es que:

“(..) no hay nada más simpático que la comunión espiritual que se establece entre profesores y alumnos. Ni existe tarea más grata que la de iniciarlos en los profundos y variados misterios de la vida universal, tales como los que conoce la ciencia. Exponer a un joven las leyes de la naturaleza, según han sido reveladas al geógrafo, al físico y al naturalista; saciar su sed de belleza dándole a conocer las

\footnotetext{
12 ÁVILA, Víctor: op. cit., pág.138.
} 
fruiciones estéticas de la literatura y de las bellas artes; ofrecerle la experiencia de los siglos mediante la exposición razonada de la historia; educar su razón y su espíritu con las disciplinas de la filosofía; iluminarlo, guiarle, aconsejarle, en una palabra, apoderarse de su entidad individual y prepararla con cariño e interés para su desenvolvimiento futuro, es, ciertamente señores, la función más augusta que puede desempeñar el hombre en el seno de la sociedad"13.

Este sentimiento lo comparten los editores de la revista que además de ser profesores en el Instituto Nacional y más tarde en la Universidad, ocuparon los más altos cargos académicos en ambas instituciones, luchando siempre por la mejora de la enseñanza y la difusión de la cultura entre una amplia capa de la población. Por otro lado, hay que resaltar su preocupación por la investigación en el terreno de la pedagogía son constantes, de ahí que en la revista encontremos trabajos de los más avanzados pedagogos del momento, además de discursos sobre la educación y ensayos filosóficos. En este número, por ejemplo, José D. Crespo publica un artículo (continuación de otro anterior) en el que intenta solucionar la disputa que existe "entre el humanismo y el naturalismo en el campo de la educación", algo que todavía hoy no se ha solucionado.

La conclusión de José D. Crespo es clara: no existe conflicto entre las ciencias y las humanidades porque ambas son indispensables y cualquier política educativa que menosprecie a una de las dos es desastrosa. Y es que para él:

13 ALFARO, Ricardo F.: Art. cit., pág.2. 
"Educar para formar meros eruditos teóricos o meros hombres de ciencia, es defraudar los intereses de la sociedad, no realizando su ideal' ${ }^{14}$.

\section{3. Una tribuna abierta.}

\section{4.}

La Revista Nueva, como el Instituto Nacional, está abierta a la discusión y no duda en publicar un artículo del anarquista español José María Blázquez de Pedro ${ }^{15}$ titulado "El sindicalismo frente a la democracia" con el que quiere "refutar ciertos errores de monta" cometidos por Cristóbal Rodríguez en otro artículo titulado "La crisis actual de la Democracia" que se publicó en el no 4 de la misma revista.

A Blázquez de Pedro le molestó especialmente que Cristóbal Rodríguez dijese:

"Unos y otros (se refiere a sindicalistas y conservadores) están contestes y se armonizan a las mil maravillas en impugnar, como una cosa estéril, torpe y perniciosa además, el individualismo, base del sistema democrático"16

El anarquista español, que será elegido unos años después miembro del Comité Central Ejecutivo de la recién fundada Federación Obrera de la república de Panamá, se enfrenta al ideólogo conservador planteando que:

"Puesto que democracia quiere decir gobierno del pueblo, el Individualismo no es, no puede ser democrático, ni aristocrático, ni monocrático, ni teocrático, ni plutocrático, sino sencilla y solamente

\footnotetext{
${ }^{14}$ CRESPO, José D.: “CONFLICTO entre el humanismo y el naturalismo en el campo de la educación por Don José D. Crespo" en La Revista Nueva Panamá, enero de 1917, p. 75.

15 Éste no es el único artículo que José María Blázquez de Pedro publica en La Revista Nueva.

${ }^{16}$ BLAZQUEZ DE PEDRO, J.M.: “El sindicalismo frente a la democracia”, La Revista Nueva, enero de 1917, pág 54
} 
acrático, o dicho con palabra más conocida por cualquiera, anarquista (...). El individuo que apetece ser libre, ampliamente libre, no se aviene a ser gobernado por el pueblo, ni por los mejores, ni por el rey, ni por el presidente, ni por los clérigos, ni por los ricos, ni por nadie; quiere gobernarse por sí mismo, y que los demás racionales se gobiernen también a sí propios, pues sabe, gracias al raciocinio y a una experiencia milenaria, que todos los gobernantes resultan y resultarán malos". ${ }^{17}$

En cuanto a que los sindicalistas desprecian el Humanitarismo y el Intelectualismo dice este revolucionario:

"Precisamente porque los sindicalistas saben sentir y pensar, porque atinan a comprender sus dolores y los de sus semejantes y se afanan por remediarlos, porque son certeros en la investigación de los motivos de los males sociales, porque alcanzan a descubrir el origen y tramitación de las injusticias reinantes, porque se elevan en el orden sensorial y mental, porque se hacen más sensibles y más conocedores, porque se humanizan y se intelectualizan (...). Si no sintieran, si no pensaran, si no fueran humanitarios e intelectuales no se asociarían, no darían constantes batallas al capital y a la autoridad unidos, no escribirían, no hablarían, no se agitarían, no propagarían por todas partes, como lo hacen a diario, con riesgo incesante de su bienestar, de su libertad y hasta de su vida." ${ }^{18}$

Y con su vida pagó sus ideas Blazquez de Pedro. Llegó a Panamá en 1914 y realizó una intensa labor como teórico, agitador y propagandista revolucionario, fue un anarquista simpatizante de la revolución socialista de 1917, fundó junto a otros

\footnotetext{
17 Ibidem, pág. 55 .

18 Ibidem. pág. 57.
} 
panameños el "Grupo Comunista" en 1921 y fue deportado a Cuba en 1925, cuando "el gobierno liberal de Rodlfo Chiari, representante característico de la vieja casta oligárquica dominante, y los inquilinos pobres agrupados en la Liga Inquilinaria"19 10 hicieron detener. Allí fue encarcelado junto a su hermano y ambos murieron en 1927 víctimas de torturas y enfermedades.

No podemos comentar las 80 páginas de este número de La Revista Nueva que llegó por casualidad a nuestras manos y nos parece apasionante porque refleja el pensamiento de algunos de los hombres que protagonizaron aquellos años. $Y$ es que quienes dirigieron la revista y quienes colaboraron en ella estaban en ese momento haciendo historia.

\footnotetext{
${ }^{19}$ ÁVILA, Víctor: op. cit. pág. 215.
} 